Biactica

\section{OCORRÊNCIA DE MALFORMAÇÕES CONGÊNITAS EM RECÉM-NASCIDOS CONCEBIDOS POR MÉTODOS DE FERTILIZAÇÃO ARTIFICIAL}

A revista The New England Journal of Medicine de 7 de março de 2002 traz dois artigos relativos ao aumento significativo de risco de ocorrência de malformações em crianças concebidas por métodos de fertilização artificial.

A observação de pesquisadores da Divisão de Saúde Reprodutiva do Centro de Tratamento de Doenças Crônicas de Atlanta mostra que RN concebidos por diferentes métodos de fertilização "in vitro" apresentaram um número desproporcionalmente elevado de crianças prematuras com muito baixo peso'.

O outro estudo foi realizado por cientistas do Instituto de Pesquisa de Saúde da Criança da Universidade do Oeste da Austrália e apresenta dados ainda mais preocupantes. Foram estudadas 301 crianças concebidas pelo método de injeção intracitoplasmática de espermatozóide, 837 através da técnica de fertilização "in vitro". O grupo controle foi constituído por 4000 crianças concebidas naturalmente. $\mathrm{O}$ estudo comparativo demonstrou incidência significativamente maior de malformações cardiovasculares, urogenitais, cromossônicas e músculo-esqueléticas ${ }^{2}$ nos RN concebidos artificialmente.

Os autores especulam sobre as causas da maior presença de malformações e sugerem como fatores que poderiam ser responsabilizados: idade avançada das mães, causas subjacentes à infertilidade, medicamentos usados para induzir a ovulação ou manter a gravidez nos estágios iniciais da gestação ou, ainda, fatores relacionados à técnica de congelamento dos embriões.

\section{Comentário}

Há que se partir do pressuposto que todo casal deva ter direito à procriação e merece receber ajuda médica, através de todos os métodos disponiveis de fertilização assistida.
Com índice de sucesso bastante modesto (inferior a 5\%) na década de 1980, passados pouco mais de 20 anos, as gravidezes "assistidas" ora bem sucedidas alcançam cifras próximas a $50 \%$ em mulheres com até 35 anos de idade. Em 25 de jultho de 2003, Louise Brown, o primeiro "bebê de proveta", comemorou com saúde, 25 anos de vida.

Do cientista, entretanto, exige-se espirito de humildade e resoluta adesão ao princípio da imparcialidade diante dos fatos produzidos pelo avanço do conhecimento. Não the é permitido restar cativo ante o fascínio da tecnociência. A propósito, Hans Jonas nos adverte em Princípio da Responsabilidade que "se nada tem tanto sucesso como o êxito, nada nos ameaça tanto como ele". Se parece óbvio que é inaceitável satanizar a ciência, insensato, também, será endeusá-la acriticamente. Dominados ainda pela supremacia da razão patrocinada pelo Iluminismo, temos que aprender a conviver com limitações do conhecimento e ordenar nossas convicçóes com equilibrio e inspiradas no sentido ético.

A resposta à questão aristotélica de como se tem que agir não será dada pela simples consulta a um programa de computador por mais bem atualizado que seja e nada substitui, neste estágio de evolução dos conhecimentos científicos, a procura do argumento razoável dentro de um debate aberto, plural e inclusivo.

Aos profissionais que trabalham com fertilização humana assistida pede-se além da prudência aristotélica, o respeito incondicional à autonomia do casal que se apresenta para receber o benefício da nova metodologia. Importante considerar que decisóes verdadeiramente autônomas somente o são, se tomadas com conhecimento pleno de todos os fatos científicos que a situação clínica impóe. Destituída de amparo moral estão as decisóes guiadas por acentuada assimetria na relação médico-paciente, omitindo o profissional, por qualquer razão que seja, a informação científica do conhecimento do casal. Como ensinou Potter, os profissionais de saúde estão obrigados a considerar não somente as decisóes clínicas cotidianas, mas também as conseqüências a longo prazo das mesmas, o que na situação que envolve o desenvolvimento normal de um ser humano recém-concebido é obviamente imprescindivel e, se assim não o for, caracterizará atitude carente de moralidade.

José Eduardo de Siqueira

Referências

I. Shieve LA, Meikle SF, Ferre C, Peterson $H B$, Jeng $G$. Low and very low birth weight in infants conceived with use of assisted reproductive technology: N Engl I Med 2002; 346(10):731-7.

2. Hansen M, Kurinczuk JJ, Bower C, Webb S. The risk of major birth defects after intracytoplasmic sperm injection and in vitro fertilization: N Engl I Med 2002; 346( I0):725-30.

\section{Clinica Ciriongiea \\ CIRURGIA BARIÁTRICA PARA ADOLESCENTES MUITO OBESOS}

A obesidade grave em adolescentes entre 12 e 18 anos é indicação para cirurgia bariátrica de acordo com o Instituto Nacional de Saúde dos Estados Unidos. A gastroplastia é o procedimento de escolha nestas situações. Foram operados 33 adolescentes entre 1981 e 200I com média de índice de massa corporal (IMC) de 52 e média de idade de 16 anos e que apresentavam comorbidades como hipertensão, diabetes, apnéia do sono, refluxo gastroesofágico, incontinência urinária, ovário policístico e asma. Não houve mortes nem fístulas pós-operatórias. Como complicações ocorreram: um caso de embolia pulmonar, infecção de ferida operatória em cinco pacientes, três estenoses de anastomoses gastrojejunais, tratadas endoscopicamente, e úlcera marginal em quatro casos. Como complicações tardias houve um caso de obstruçãa de intestino delgado e seis hérnias incisionais.

Ocorreu perda substancial de peso nestes pacientes e as comorbidades desapareceram um ano após a cirurgia. Houve retorno à vida normal, ressocialização dos pacientes com conclusão de cursos secundários e universitários.

Os autores concluíram que a cirurgia bariátrica em adolescentes é segura, havendo perda significante de peso com remissão das comorbidades, e promove a ressocialização dos operados. 


\section{Comentário}

A obesidade em adolescentes em nosso meio e no mundo tem aumentado em proporção assustadora, trazendo assim um grande número de doenças associadas que não se viam nessa faixa etária. Isso se deve principalmente às mudanças de hábitos alimentares e ao sedentarismo. Nos casos em que o emagrecimento não é conseguido pelo tratamento clínico, deve-se considerar a indicação do tratamento operatório por ser eficiente, seguro e causar a remissão rápida da obesidade e das suas comorbidades.

\section{Elias Jirjoss Ilias Osvaldo Antonio Prado Castro Paulo Kassab}

Referência

I. Sugerman HJ, Sugerman EL, DeMaria EJ, Kellum JM, Kennedy C, Mowery Y, et al. Bariatric surgery for severely obese adolecents. I Gastrointest Surg 2003; $7: 102-7$

\section{Climica Wedica INSUFICIÊNCIA RENAL CRÔNICA EM TRANSPLANTE DE ÓRGÃO NÄO RENAL}

O transplante de órgãos representa hoje a melhor terapia substitutiva para pacientes com doença crônica terminal. Apesar do rim ser o órgão mais transplantado no país, seguido dos enxertos de córnea, os transplantes de outros órgãos sólidos têm crescido gradativamente nos últimos anos, exemplificando os transplantes de pâncreas (duplo ou não), figado e coração.

A rejeição aguda ainda persiste como a principal causa de perda precoce no transplante de órgãos, apesar de sua incidência ter diminuído com o advento de novos potentes imunossupressores. A longo prazo, a maioria dos órgãos serão perdidos em conseqüência de um processo crônico fibrótico irreversível, a rejeição crônica. Com o aumento da sobrevida dos pacientes, vários autores vêm demonstrando a prevalência e importância de complicações imunológicas ou não tardias, muitas delas secundárias ao uso dos imunossupressores, representando um importante fator de risco para perda do enxerto.

Recentemente, Ojo et al. relataram a incidência de insuficiência renal crônica em pacientes submetidos a transplante de órgãos sólidos que não rim. Os autores analisaram 69.321 receptores de transplante de órgãos sólidos, excluído rim, num período de 1990 a 2000 no Estados Unidos. Foram estudadas a incidência cumulativa de insuficiência renal crônica (IRC) e as variáveis clínicas relacionadas. Em um período de 36 meses de acompanhamento, 16,5\% dos pacientes desenvolveram IRC, e $28,9 \%$ deles necessitaram de diálise. O risco de desenvolver IRC em cinco anos foi maior nos receptores de transplante intestinal, seguidos dos receptores de fígado, pulmão, coração e finalmente coração-pulmão. Entre os fatores relacionados a um maior risco de IRC foram encontrados a idade avançada $(R R=1.36$, para incrementos de 10 anos), sexo feminino $(R R=0.74)$, diabetes mellitus ( $R R=1.42)$, hipertensão arterial $(R R=1.18)$, insuficiência renal aguda no pós-operatório $(R R=2.13)$, sorologia positiva para hepatite $C(R R=1.15)$, todos com significância estatística. A presença de IRC pós-transplante de órgão sólido que não rim foi associada a um risco significativamente aumentado de morte ( $R R=4.55, p<0.001$ ). $O$ transplante renal nos pacientes com IRC foi associado com uma queda no risco de morte quando comparada à terapia dialítica $(R R=0.56, P=0.02)$.

\section{Comentário}

O transplante de órgãos sólidos representa a melhor opção terapêutica, considerando custos e melhorias na qualidade de vida dada ao paciente. Com o uso corriqueiro dos novos imunossupressores e o crescente número de cirurgias realizadas, o manuseio clínico do paciente transformou-se na etapa mais importante para se garantir a sobrevida a longo prazo dos enxertos. Mesmo assim, complicações crônicas tendem a ser mais freqüentes, com implicações na sobrevida do enxerto e do paciente.

Neste estudo pioneiro, a incidência de IRC foi aferida em uma grande população de transplantados, e demonstrado seu impacto na sobrevida nos mesmos. A IRC foi mais freqüente em alguns tipos específicos de transplante, incluindo o transplante de intestino, cuja alta imunogenicidade ocasiona o uso maior de drogas imunossupressoras nefrotóxicas, e o de figado, que apresenta uma maior incidência de insuficiência renal aguda pré-transplante. De forma importante, os resultados deste trabalho mostram que a presença de IRC aumenta o risco de morte nos transplantados, e que o transplante renal é a melhor opção substitutiva renal. A indicação de diálise nestes pacientes relaciona-se com pior sobrevida.

Especificamente, não existem métodos laboratoriais não-invasivos que possam detectar quais pacientes estão sob risco aumentado de lesão renal. A deteç̧ão precoce de dano renal reversível não pode ser feita pela simples análise das escorias séricas. Recentemente, foi demonstrado que pacientes sob risco aumentado de lesão fibrótica renal podem ser detectados através da dosagem urinária da proteína ligadora do retinol (RBP). Pacientes transplantados cardíacos com elevados níveis de RBP urinários, provavelmente secundário a nefrotoxicidade dos imunossupressores, apresentam elevado risco de IRC. A RBP é uma proteína de baixo peso molecular presente na urina em pacientes com dano túbulointersticial e apresenta correlação positiva com perda renal a longo prazo. A determinação rotineira da RBP urinária pode ajudar no manuseio clínico diário de transplantados sob risco aumentado de insuficiência renal.

\section{Niels Olsen Saraiva Câmara Álvaro Pacheco-Silva}

\section{Referências}

I. Ojo OA, Held PJ, Port FK, Wolfe RA, Leichtman $A B$, Young EW, et al. Chronic renal failure after transplantation of a nonrenal organ. N Engl J Med 2003; 349:931-40.

2. Camara NO, Matos AC, Rodrigues DA, Pereira $A B$, Pacheco-Silva A. Early detection of heart transplant patients with increased risk of ciclosporin nephrotoxicity. Lancet 2001; 357:856-7. 